\title{
AN HNN-EXTENSION WITH CYCLIC ASSOCIATED SUBGROUPS AND WITH UNSOLVABLE CONJUGACY PROBLEM
}

\author{
JODY MEYER LOCKHART
}

Dedicated to memory of William Werner Boone

\begin{abstract}
In this paper, we consider the conjugacy problem for HNNextensions of groups with solvable conjugacy problem for which the associated subgroups are cyclic. An example of such a group with unsolvable conjugacy problem is constructed. A similar construction is given for free products with amalgamation.
\end{abstract}

\section{INTRODUCTION}

It is known that an HNN-extension of a group with solvable conjugacy problem may have unsolvable conjugacy problem. Some restrictions placed on the type of HNN-extensions force the conjugacy problem to be solvable. In this paper, we investigate $\mathrm{HNN}$-extensions with infinite cyclic associated subgroups. That is, we consider HNN-extensions of the form

$$
G=\left\langle H, t ; t^{-1} a t=b\right\rangle,
$$

where $a, b \in H$ are of infinite order. We also consider the analogous situation of free products with cyclic amalgamated subgroups.

The conjugacy problem for HNN-extensions and free products with amalgamation of this type has been considered by M. Anshel and P. Stebe [1], L. P. Comerford and B. Truffault [2], R. D. Hurwitz [3], and S. Lipschutz [4], among others. All obtained results giving conditions which guarantee that such groups have solvable conjugacy problem.

In this paper, we approach the problem from the opposite direction. An example of an HNN-extension $G=\left\langle H, t ; t^{-1} a t=b\right\rangle$ where $H$ has solvable conjugacy problem, $G$ has solvable word problem, but $G$ has unsolvable conjugacy problem is constructed. A similar example is given involving free products.

In $\S 2$, we show the existence of a one-to-one recursive function $f: N \rightarrow N$ with nonrecursive range such that $S_{n}=\{f(k n): k=1,2, \ldots\}$ is recursive for

Received by the editors January 4, 1988.

1980 Mathematics Subject Classification (1985 Revision). Primary 03D40, 20F05, 20F10; Secondary 03020. 
each $n=2,3, \ldots$. In $\S 3$, we use the function $f$ to construct group presentations $H$ and $G$, each with a recursive set of generators and a recursively enumerable set of defining relators. $H$ has the following properties.

(i) $H$ has solvable conjugacy problem;

(ii) $H$ has an infinite cycle subgroup $\langle d\rangle$ such that

(a) there is no algorithm to decide if an arbitrary $W$ in $H$ is conjugate to an element of $\langle d\rangle$;

(b) the problem of membership in $\langle d\rangle$ is decidable;

(iii) If $W \in H$ then $W$ is not conjugate in $H$ to $W^{-1}$.

Let $G=\left\langle H, t ; t^{-1} d t=d^{-1}\right\rangle$. The group $G$ has solvable word problem by (ii)(b). Straightforward arguments show that for any $W \in H, W$ is conjugate to $W^{-1}$ in $G$ if and only if $W$ is conjugate in $H$ to an element of $\langle d\rangle$. Thus $G$ has unsolvable conjugacy problem.

We then use the standard HNN embedding into a two-generator group to obtain the result for recursively presented groups. Free products with amalgamation are also considered.

$\S 4$ contains the proofs of some assertions used but not proved in $\S 3$.

\section{The FUNCTION $f$}

In this section, we prove the existence of a recursive function $f$ with certain special properties.

Lemma 1. There is a one-to-one recursive function $f: N \rightarrow N$ with nonrecursive range such that $S_{n}=\{f(k n): k=1,2, \ldots\}$ is recursive for each $n=2,3,4, \ldots$.

Proof. Let $g$ be any one-to-one recursive function with nonrecursive range. Let $p_{j}$ be the $j$ th prime number. Define $f$ by

$$
f(1)=1, f\left(p_{i_{1}}^{\alpha_{1}} p_{i_{2}}^{\alpha_{2}} \cdots p_{i_{n}}^{\alpha_{n}}\right)=p_{g\left(i_{1}\right)}^{\alpha_{1}} p_{g\left(i_{1}\right)+i_{2}}^{\alpha_{2}} p_{g\left(i_{1}\right)+i_{3}}^{\alpha_{3}} \cdots p_{g\left(i_{1}\right)+i_{n}}^{\alpha_{n}}
$$

where $i_{1}<i_{2}<\cdots<i_{n}$. The function $f$ is clearly recursive.

We next show that $f$ is one-to-one. Suppose

$$
f\left(p_{i_{1}}^{\alpha_{1}} p_{i_{2}}^{\alpha_{2}} \cdots p_{i_{n}}^{\alpha_{n}}\right)=f\left(p_{j_{1}}^{\beta_{1}} p_{j_{2}}^{\beta_{2}} \cdots p_{j_{m}}^{\beta_{m}}\right) .
$$

Then

$$
p_{g\left(i_{1}\right)}^{\alpha_{1}} p_{g\left(i_{1}\right)+i_{2}}^{\alpha_{2}} \cdots p_{g\left(i_{1}\right)+i_{n}}^{\alpha_{n}}=p_{g\left(j_{1}\right)}^{\beta_{1}} p_{g\left(j_{1}\right)+j_{2}}^{\beta_{2}} \cdots p_{g\left(j_{1}\right)+j_{m}}^{\beta_{m}} \text {. }
$$

Since $g\left(i_{1}\right)<g\left(i_{1}\right)+i_{2}<g\left(i_{1}\right)+i_{3}<\cdots<g\left(i_{1}\right)+i_{n}$ and

$$
g\left(j_{1}\right)<g\left(j_{1}\right)+j_{2}<g\left(j_{1}\right)+j_{3}<\cdots<g\left(j_{1}\right)+j_{m},
$$

it follows that

$$
g\left(i_{1}\right)=g\left(j_{1}\right), \quad g\left(i_{1}\right)+i_{2}=g\left(j_{1}\right)+j_{2}, \ldots, g\left(i_{1}\right)+i_{n}=g\left(j_{1}\right)+j_{n} .
$$

Therefore, $m=n$ and $i_{k}=j_{k}$ and $\alpha_{k}=\beta_{k}$ for all $k=1, \ldots, n$. Hence,

$$
p_{i_{1}}^{\alpha_{1}} p_{i_{2}}^{\alpha_{2}} \cdots p_{i_{n}}^{\alpha_{n}}=p_{j_{1}}^{\beta_{1}} p_{j_{2}}^{\beta_{2}} \cdots p_{j_{m}}^{\beta_{m}} \text {. }
$$


Now consider the range of $f$.

$$
p_{i} \in \text { range } f\left\{\begin{array}{l}
\text { iff } p_{i}=f\left(p_{j}\right) \text { for some } j, \\
\text { iff } p_{i}=p_{g(j)} \text { for some } j, \\
\text { iff } i \in \text { range } g .
\end{array}\right.
$$

Since the range of $g$ is nonrecursive, the range of $f$ is nonrecursive.

Next consider $S_{n}$ where $n$ is prime. We claim that

if and only if

$$
p_{j_{1}}^{\alpha_{1}} p_{j_{2}}^{\alpha_{2}} \cdots p_{j_{l}}^{\alpha_{l}} \in S_{p_{i}}
$$

(i) $j_{1}=g(i)$ and $j_{l}>j_{l-1}>\cdots>j_{2}>j_{1}+i$; or

(ii) $j_{1}=g\left(i^{\prime}\right)$ for some $i^{\prime}\left\langle i, j_{l}>\cdots>j_{2}>j_{1}+i^{\prime}\right.$, and $j_{s}-j_{1}=i$ for some $s$ between 2 and $l$.

It follows immediately from the claim that $S_{n}$ is recursive if $n$ is prime.

We now prove the claim. First assume that $p_{j_{1}}^{\alpha_{1}} p_{j_{2}}^{\alpha_{2}} \cdots p_{j_{l}}^{\alpha_{l}}$ is in $S_{p_{i}}$. Then

$$
p_{j_{1}}^{\alpha_{1}} p_{j_{2}}^{\alpha_{2}} \cdots p_{j_{l}}^{\alpha_{l}}=f\left(p_{i_{1}}^{\beta_{1}} p_{i_{2}}^{\beta_{2}} \cdots p_{i_{l}}^{\beta_{l}}\right)
$$

for some $p_{i_{1}}^{\beta_{1}} p_{i_{2}}^{\beta_{2}} \cdots p_{i_{1}}^{\beta_{1}}$ where $i_{1}<i_{2}<\cdots<i_{l}$ and $i=i_{t}$ for some $t=$ $1, \ldots, l$. There are two cases to consider: (1) $t=1$ and (2) $t \geq 2$.

Case (1). If

then

$$
p_{j_{1}}^{\alpha_{1}} p_{j_{2}}^{\alpha_{2}} \cdots p_{j_{l}}^{\alpha_{l}}=f\left(p_{i}^{\beta_{1}} p_{i_{2}}^{\beta_{2}} \cdots p_{i_{l}}^{\beta_{l}}\right)
$$

$$
p_{j_{1}}^{\alpha_{1}} p_{j_{2}}^{\alpha_{2}} \cdots p_{j_{l}}^{\alpha_{l}}=p_{g(i)}^{\beta_{1}} p_{g(i)+i_{2}}^{\beta_{2}} \cdots p_{g(i)+i_{l}}^{\beta_{l}}
$$

Therefore, $j_{1}=g(i)$ and $j_{2}>j_{1}+i$; that is, (i) holds.

Case (2). If

then

$$
p_{j_{1}}^{\alpha_{1}} p_{j_{2}}^{\alpha_{2}} \cdots p_{j_{l}}^{\alpha_{l}}=f\left(p_{i_{1}}^{\beta_{1}} p_{i_{2}}^{\beta_{2}} \cdots p_{i}^{\beta_{l}} \cdots p_{i_{l}}^{\beta_{l}}\right),
$$

$$
p_{j_{1}}^{\alpha_{1}} p_{j_{2}}^{\alpha_{2}} \cdots p_{j_{l}}^{\alpha_{l}}=p_{g\left(i_{1}\right)}^{\beta_{1}} p_{g\left(i_{1}\right)+i_{2}}^{\beta_{2}} \cdots p_{g\left(i_{1}\right)+i}^{\beta_{l}} \cdots p_{g\left(i_{1}\right)+i_{l}}^{\beta_{l}} .
$$

Therefore, $j_{1}=g\left(i_{1}\right)$ for some $i_{1}<i, j_{2}>j_{1}+i_{1}$, and $i=j_{t}-j_{1}$ for some $t$ between 2 and $l$. That is, (ii) holds.

Now suppose $n=p_{j_{1}}^{\alpha_{1}} p_{j_{2}}^{\alpha_{2}} \cdots p_{j_{l}}^{\alpha_{l}}$ satisfies (i) or (ii). We will show that $n \in S_{p_{i}}$.

Suppose $n$ satisfies (i). Let $t_{m}=j_{m}-j_{1}$ for $m=2, \ldots, l$. Therefore, (i) gives $t_{l}>t_{l-1}>\cdots>t_{2}>i$. We have

$$
\begin{aligned}
f\left(p_{i}^{\alpha_{1}} p_{t_{2}}^{\alpha_{2}} p_{t_{3}}^{\alpha_{3}} \cdots p_{t_{l}}^{\alpha_{l}}\right) & =p_{g(i)}^{\alpha_{1}} p_{g(i)+t_{2}}^{\alpha_{2}} p_{g(i)+t_{3}}^{\alpha_{3}} \cdots p_{g(i)+t_{l}}^{\alpha_{l}} \\
& =p_{j_{1}}^{\alpha_{1}} p_{j_{2}}^{\alpha_{2}} p_{j_{3}}^{\alpha_{3}} \cdots p_{j_{l}}^{\alpha_{l}} .
\end{aligned}
$$

Hence, $n \in S_{p_{i}}$.

Suppose $n$ satisfies (ii). Let $t_{m}=j_{m}-j_{1}$ for $m=2,3, \ldots, l$. Then $t_{l}>t_{l-1}>\cdots>t_{3}>t_{2}>i^{\prime}$ where $g\left(i^{\prime}\right)=j_{1}$. Therefore,

$$
\begin{aligned}
f\left(p_{i^{\prime}}^{\alpha_{1}} p_{t_{2}}^{\alpha_{2}} p_{t_{3}}^{\alpha_{3}} \cdots p_{t_{l}}^{\alpha_{l}}\right) & =p_{g\left(i^{\prime}\right)}^{\alpha_{1}} p_{g\left(i^{\prime}\right)+t_{2}}^{\alpha_{2}} \cdots p_{g\left(i^{\prime}\right)+t_{l}}^{\alpha_{l}} \\
& =p_{j_{1}}^{\alpha_{1}} p_{j_{2}}^{\alpha_{2}} \cdots p_{j_{l}}^{\alpha_{l}}
\end{aligned}
$$


and since $j_{s}-j_{1}=i$ for some $s$, we have $t_{s}=i$ for some $s$. Therefore, $n \in S_{p_{i}}$. This completes the proof of the claim.

To complete the proof of the lemma, we need to show that $S_{n}$ is recursive if $n>1$ and $n$ is not prime. Let $p$ be any prime divisor of $n$. Then, $x \in S_{n}$ implies $x \in S_{p}$. To determine if $x \in S_{n}$, first determine if $x \in S_{p}$. If not, then $x \notin S_{n}$. If so, we can effectively find the unique number $a$ such that $x=f(a p)$. If $n$ divides $a p$ then $x \in S_{n}$. Otherwise, $x \notin S_{n}$.

\section{THE GROUPS}

In this section, we give an example of a recursive group presentation $N$ with solvable conjugacy problem and with infinite cyclic subgroups $A_{1}$ and $A_{2}$ such that the HNN-extension of $G$ associating $A_{1}$ and $A_{2}$ has unsolvable conjugacy problem.

If $W_{1}$ and $W_{2}$ are words, we use $W_{1} \equiv W_{2}$ to mean that $W_{1}$ and $W_{2}$ are identical words, $W_{1}=W_{2}$ to mean that $W_{1}$ and $W_{2}$ are equal as elements of the group $G, W_{1} \underset{F r}{=} W_{2}$ to mean that $W_{1}$ and $W_{2}$ are freely equal, and $W_{1} \underset{G}{\sim} W_{2}$ to mean that $W_{1}$ and $W_{2}$ are conjugate as elements of $G$.

Let $f$ be the function of Lemma 1 and let

$$
H=\left\langle x_{1}, z_{1}, x_{2}, z_{2}, \ldots ; z_{i}^{-1} x_{f(i)} z_{i}=z_{1}^{-1} x_{1}^{i} z_{1} ;(i=2,3,4, \ldots)\right\rangle .
$$

We begin with some definitions.

Definition. If $W \equiv x_{i_{1}}^{\alpha_{1}} z_{j_{1}}^{\beta_{1}} \cdots x_{i_{n}}^{\alpha_{n}} z_{j_{n}}^{\beta_{n}}$, where $\alpha_{k}, \beta_{k}$ are integers, then $W$ is said to be in condensed form if $\beta_{k}=0$ implies $i_{k} \neq i_{k+1}$ and $\alpha_{k}=0$ implies $j_{k-1} \neq j_{k}$.

Definition. If $W \equiv x_{i_{1}}^{\alpha_{1}} z_{j_{1}}^{\beta_{1}} \cdots x_{i_{n}}^{\alpha_{n}} z_{j_{n}}^{\beta_{n}}$, where $\alpha_{k}, \beta_{k}$ are integers, then $W$ is said to be reduced if

(i) $W$ is freely reduced;

(ii) $i_{s} \neq f\left(j_{s-1}\right)$ for $s=2,3, \ldots, n, i_{s} \neq f\left(j_{s}\right)$ for $s=1,2, \ldots, n$ and $i_{1} \neq f\left(j_{n}\right) ;$ and

(iii) no nontrivial subword of $W$ or of any cyclic permutation of $W$ equals 1 in $H$.

Notice that the free group presented by

$$
B=\left\langle x_{j}(j \notin \text { range } f), x_{1}, z_{1}, z_{2}, \ldots ;\right\rangle
$$

is isomorphic to the group presented by $H$.

We first show that $H$ has solvable word problem from which it follows that there is an effective procedure to determine if a word is reduced.

Lemma 2. $H$ has solvable word problem.

Proof. Assume $W \equiv x_{i_{1}}^{\alpha_{1}} z_{j_{1}}^{\beta_{1}} \cdots x_{i_{n}}^{\alpha_{n}} z_{j_{n}}^{\beta_{n}}$ is freely reduced and in condensed form. If $i_{1}, \ldots, i_{n}$ are not in the range of $f$, then $W=\frac{\bar{H}}{1}$ if and only if $W=1$ 
generality, that $\sigma^{\prime} \neq 1$. By Lemma $1, S_{\sigma^{\prime}}$ is recursive. Determine if $i \in S_{\sigma^{\prime}}$. If not, then (ii) is not satisfied. If so, effectively find $a=m \sigma^{\prime}$ with $i=f(a)$. The only possible value for $b$ then is $(a \alpha) / \sigma$. If $j=f(a \alpha / \sigma)$, then (ii) is satisfied. Otherwise, (ii) is not satisfied.

Theorem 1. There is a group presentation $H$ with a recursive set of generators and a recursively enumerable set of defining relators with solvable conjugacy problem and an $H N N$-extension $G$ of $H$ associating infinite cyclic subgroups of $H$ that has unsolvable conjugacy problem.

Proof. Let $H=\left\langle x_{1}, z_{1}, x_{2}, z_{2}, \ldots ; z_{i}^{-1} x_{f(i)} z_{i}=z_{1}^{-1} x_{1}^{i} z_{1} \quad(i=2,3, \ldots)\right\rangle$ be the group of Lemma 4 and let

$$
G=\left\langle H, t ; t^{-1} z_{1}^{-1} x_{1} z_{1} t=z_{1}^{-1} x_{1}^{-1} z_{1}\right\rangle .
$$

Note that $z_{1}^{-1} x_{1} z_{1}$ has infinite order. We claim that $x_{j} \tilde{G}_{j}^{-1}$ if and only if $j \in$ range of $f$, from which it immediately follows that $G$ has unsolvable conjugacy problem.

We now prove the claim. If $j \in$ range of $f$, then in $G$

$$
\begin{aligned}
x_{j} & \equiv x_{f(n)}=z_{n} z_{1}^{-1} x_{1}^{n} z_{1} z_{n}^{-1} \sim z_{1}^{-1} x_{1}^{n} z_{1} \sim z_{1}^{-1} x_{1}^{-n} z_{1} \\
& \sim z_{n} z_{1}^{-1} x_{1}^{-n} z_{1} z_{n}^{-1}=x_{f(n)}^{-1} \equiv x_{j}^{-1} .
\end{aligned}
$$

If $x_{j} \widetilde{G}_{\sigma_{j}} x^{-1}$, then using the facts that $x_{j}, x_{j}^{-1} \in H$ and $G$ is an HNNextension of $H$, either

(i) $x_{j} \widetilde{\sim}_{H} x_{j}^{-1}$, or

(ii) there is a sequence of words $V_{1}, V_{2}, \ldots, V_{2 p}$ with $x_{j} \equiv V_{1}$ and $x_{j}^{-1} \equiv$ $V_{2 p}$ such that $V_{2 j+1} \underset{H}{\sim} V_{2 j+2}$ for $j=0, \ldots, p-1$ and, for each $j=$ $1, \ldots, p-1$, there is an $\varepsilon= \pm 1$ with $t^{-\varepsilon} V_{2 j} t^{\varepsilon}=V_{2 j+1}$.

We will first show that (i) is impossible. If $j \notin$ range $f$, then $x_{j} \underset{H}{\sim} x_{j}^{-1}$ if and only if $x_{j} \underset{F r}{\widetilde{F}_{j}} x_{j}^{-1}$ which is clearly impossible. If $j=f(n)$, then

$$
x_{j} \widetilde{H}_{j} x_{j}^{-1}\left\{\begin{array}{l}
\text { iff } z_{n} z_{1}^{-1} x_{1}^{n} z_{1} z_{n}^{-1} \widetilde{F r}_{n} z_{n} z_{1}^{-1} x_{1}^{-n} z_{1} z_{n}^{-1}, \\
\text { iff } x_{1}^{n} \widetilde{F r}_{1} x_{1}^{-n}
\end{array}\right.
$$

which is impossible since $n \neq 0$. Therefore, if $x_{j} \underset{G}{\widetilde{x}_{j}^{-1}}$, (ii) must occur and so $x_{j}$ must be conjugate in $H$ to an element of the form $z_{1}^{-1} x_{1}^{n} z_{1}$ where $n$ is some integer. If $j \notin$ range $f$ and $x_{j} \underset{H}{\widetilde{H}_{1}} z_{1}^{-1} x_{1}^{n} z_{1}$, then $x_{j} \underset{F r}{{ }_{F}} z_{1}^{-1} x_{1}^{n} z_{1}$ which is impossible. Therefore, $x_{j} \underset{G}{\sim_{j}^{-1}}$ implies that $j \in$ range $f$.

We mention without proof that $G$ has solvable word problem.

We now extend the above result to recursive presentations by using the standard HNN embedding into a two-generator group and checking that the appropriate properties are preserved. 
Lemma 5. Let $H=\left\langle h_{1}, h_{2}, \ldots ; s_{1}, s_{2}, \ldots\right\rangle$ be a presentation with a recursive set of generators and a recursively enumerable set of defining relators such that

(i) $H$ has solvable conjugacy problem;

(ii) $h_{i} \neq 1$ for all $i=1,2,3, \ldots$;

(iii) $h_{i} \neq h_{j}$ for all $i \neq j, i, j=1,2,3, \ldots$; and

(iv) there is an algorithm which given a word $W$ of $H$ decides if $W=h_{i}^{\varepsilon} h_{j}^{\delta}$ for some $i, j=1,2, \ldots$ and some $\varepsilon, \delta= \pm 1$ or 0 and, if so, which ones.

Let $F=H^{*}\langle a, b\rangle$ and

$$
N=\left\langle F, t ; t^{-1} a t=b, t^{-1} b^{-i} a b^{i} t=h_{i} a^{-i} b a^{i}(i=1,2, \ldots)\right\rangle .
$$

Then $N$ has solvable conjugacy problem.

Proof. It is known that $N$ has solvable word problem. Let $G_{1}$ be the group generated by $\left\{a, b^{-i} a b^{i}(i=1,2, \ldots)\right\}$ and let $G_{2}$ be the group generated by $\left\{b, h_{i} a^{-i} b a^{i}(i=1,2, \ldots)\right\}$. Let $\phi$ be the isomorphism from $G_{1}$ to $G_{2}$ defined by $\phi(a)=b$ and $\phi\left(b^{-i} a b^{i}\right)=h_{i} a^{-i} b a^{i}$ for $i=1,2, \ldots$. We need the following assertions whose proofs will be delayed until $\S 4$.

(1) There is an algorithm to decide if elements of $G_{1} \cup G_{2}$ are conjugate in $N$.

(2) There is an algorithm to decide, given $V \in F$, if $V$ is conjugate in $F$ to an element of $G_{1} \cup G_{2}$ and, if so, to find such an element.

(3) There is an effective procedure to determine, for arbitrary $X, Y \in F$, if there is a $Z \in G_{2}$ such that $X Z Y \in G_{1}$ and, if so, to produce the finite number of possible $Z$.

(4) If $X, Y \in F-G_{2}, D \in G_{2}$ is reduced, and $X D Y \in G_{2}$, then $\#_{b}(D) \leq$ $\#_{b}(X)+\#_{b}(Y)+2$, where $\#_{b}(A)$ is the number of occurrences of the symbol $b$ in the word $A$.

Since $t$-reduction is effective, we may assume that we are dealing with cyclically $t$-reduced words and we may consider the base and nonbase cases separately.

Base Case. We first consider conjugacy in $N$ of elements of $F$. Suppose $U, V \in F$. By Collins' Lemma, $U \underset{N}{\sim} V$ if and only if

(a) $U \underset{F}{\sim} V$; or

(b) there exist words $W_{1}, \ldots, W_{k}$ such that $U \underset{F}{\sim} W_{1} \underset{t}{\sim} W_{2} \underset{F}{\sim} W_{3} \sim \cdots \sim$ $W_{k} \sim \underset{F}{V}$ where $W_{1}, \ldots, W_{k} \in G_{1} \cup G_{2}$ and $\sim$ indicates conjugation by $t$ or $t^{-1}$.

Case (a) can be decided since $F$ has solvable conjugacy problem. Case (b) holds if and only if there exist $W_{1}, W_{k} \in G_{1} \cup G_{2}$ such that $U \sim{ }_{F} W_{1}$, $V \underset{F}{\sim} W_{k}$, and $W_{1} \underset{G}{\sim} W_{k}$. By assertions (1) and (2) this can be decided. 
Nonbase Case. We now consider conjugacy in $N$ of elements of $N-F$. Assume that

$$
U \equiv x_{1} t^{\varepsilon_{1}} \cdots x_{m} t^{\varepsilon_{m}} x_{m+1} \quad \text { and } V \equiv y_{1} t^{\varepsilon_{1}} \cdots y_{m} t^{\varepsilon_{m}} y_{m+1}
$$

both cyclically $t$-reduced. We consider three cases:

(i) $m=1$;

(ii) $m>1$ and the $\varepsilon_{i}$ do not cyclically alternate; i.e. $\varepsilon_{m}=\varepsilon_{1}$ or there is an $i, 1 \leq i \leq m-1$, such that $\varepsilon_{i}=\varepsilon_{i+1}$;

(iii) $m>1$ and the $\varepsilon_{i}$ do cyclically alternate.

First consider (i); $m=1$. By Collins' Lemma, we may assume that $U \equiv x t$; $V \equiv y t$; and $U \underset{N}{\sim} V$ if and only if there is a $C \in G_{2}$ such that $C^{-1} x t C \underset{N}{=} y t$. But $C^{-1} x t C \underset{N}{=} y t$ implies that $t^{-1} y^{-1} C^{-1} x t C \underset{N}{=} 1$. Therefore, $x$ and $y \in F$, $C^{-1} \in G_{2}$, and $y^{-1} C^{-1} x \in G_{1}$. By assertion (3), there are only finitely many possible $C$ and they can be effectively found. Since $N$ has solvable word problem, $C^{-1} x t C \underset{\bar{N}}{\bar{N}} y t$ can be effectively tested for each possible $C$ and so $U \sim \widetilde{N}$ can be decided in case (i).

Now consider (ii); $m>1$ and the $\varepsilon_{i}$ do not cyclically alternate. We may, by Collins' Lemma and by using $U^{-1}$ and $V^{-1}$ instead of $U$ and $V$ if necessary, assume that $\varepsilon_{m-1}=\varepsilon_{m}=-1$ and $x_{m+1}=y_{m+1}=1$. Then $U \sim \sim_{N} V$ if and only if there is a $C \in G_{1}$ such that

$$
C^{-1} x_{1} t^{\varepsilon_{1}} \cdots x_{m-1} t^{-1} x_{m} t^{-1} C t y_{m}^{-1} t y_{m-1}^{-1} \cdots t^{-\varepsilon_{1}} y_{1}^{-1}=1
$$

By considering the first two $t$-pinches on the left-hand side of $(*)$, one obtains that $C^{-1} U C \underset{\bar{N}}{=} V$ implies $x_{m} \phi(C) y_{m}^{-1} \in G_{1}$. Therefore, any conjugating element $C$ has the properties

(a) $\phi(C) \in G_{2}$ and (b) $x_{m} \phi(C) y_{m}^{-1} \in G_{1}$.

By assertion (3), there is an effective procedure that will either determine that no such $C$ exists or produce a finite list containing all $C$ satisfying (a) and (b). As in case (i), $U \underset{N}{\sim} V$ can be decided.

Now consider (iii); the $\varepsilon_{i}$ cyclically alternate. We may, without loss of generality, assume that

$$
\begin{aligned}
& U \equiv x_{1} t x_{2} t^{-1} x_{3} t x_{4} t^{-1} \cdots x_{n-1} t x_{n} t^{-1} \\
& V \equiv y_{1} t y_{2} t^{-1} y_{3} t y_{4} t^{-1} \cdots y_{n-1} t y_{n} t^{-1}
\end{aligned}
$$

$n$ is even; and $U \underset{N}{\sim} V$ if and only if there is a $C \in G_{1}$ such that $C^{-1} U C \overline{\bar{N}} V$.

Define the homomorphism $\rho: F \rightarrow\langle a, b\rangle$ by $\rho(h)=1$ for all $h \in H$, $\rho(a)=a$ and $\rho(b)=b$. Define the homomorphism $\tau: F \rightarrow F$ by $\tau(h)=h$ 
for all $h \in H, \tau(a)=b$, and $\tau(b)=a$. Now,

$$
\begin{aligned}
& C^{-1} U C_{\bar{N}}^{=} V \Rightarrow C^{-1} x_{1} t x_{2} t^{-1} \cdots x_{n} t^{-1} C t y_{n}^{-1} t^{-1} y_{n-1}^{-1} \cdots t^{-1} y_{1}^{-1}=1 \\
& \Rightarrow \\
& C^{-1} x_{1} \phi^{-1}\left(x_{2} \phi\left(x_{3} \cdots \phi\left(x_{n-1} \phi^{-1}\left(x_{n} \phi(C) y_{n}^{-1}\right) y_{n-1}^{-1} \cdots y_{3}^{-1}\right) y_{2}^{-1}\right) y_{1}^{-1}=1\right. \\
& \Rightarrow \\
& \rho\left(c^{-1} x_{1} \phi^{-1}\left(x_{2} \phi\left(x_{3} \cdots \phi\left(x_{n-1} \phi^{-1}\left(x_{n} \phi(c) y_{n}^{-1}\right) y_{n-1}^{-1} \cdots y_{3}^{-1}\right) y_{2}^{-1}\right) y_{1}^{-1}\right) \underset{\langle a, b\rangle}{=1}\right. \\
& \Rightarrow \\
& C^{-1} U_{1} C \underset{\langle a, b\rangle}{=} U_{2} \text {, where } U_{1}=\rho\left(x_{1}\right)(\tau \circ \rho)\left(x_{2}\right) \rho\left(x_{3}\right)(\tau \circ \rho)\left(x_{4}\right) \cdots(\tau \circ \rho)\left(x_{n}\right) \\
& \text { and } U_{2}=\rho\left(y_{1}\right)(\tau \circ \rho)\left(y_{2}\right) \rho\left(y_{3}\right)(\tau \circ \rho)\left(y_{4}\right) \cdots(\tau \circ \rho)\left(y_{n}\right) \\
& \Rightarrow
\end{aligned}
$$

Since such $W$ and $D$ can be effectively found, it remains only to find a bound for $m$. For then we will have effectively found the finite number of possible conjugating elements and each can be tested. We know that $x_{n}, y_{n} \notin G_{2}$ for otherwise $U, V$ would not be reduced. If $C^{-1} U C=V$, then $\phi(C) \in G_{2}$ and $x_{n} \phi(C) y_{n}^{-1} \in G_{2}$. By assertion $(4), \#_{b}(\phi(C)) \leq \#_{b}\left(x_{n}\right)+\#_{b}\left(y_{n}\right)+2$. Hence, a bound for $m$ can be found completing the proof for case (iii).

Lemma 6. Let $H$ and $N$ be as in Lemma 5 and suppose $G=\left\langle H, v ; v^{-1} d v=\right.$ $e)$ has solvable conjugacy problem where $d$ and $e$ are elements of $H$ of infinite order. Let $L=\left\langle N, v ; v^{-1} d v=e\right\rangle$. Then $L$ has unsolvable conjugacy problem. Proof. We first show that, for $y_{1}, y_{2} \in H, y_{1} \underset{H}{\sim} y_{2}$ if and only if $y_{1} \underset{N}{{ }_{N}} y_{2}$. The statement is clear from left to right. If $y_{1} \underset{N}{\sim_{2}} y_{2}$, then either

(1) $y_{1} \sim y_{2}$ or

(2) $y_{1} \underset{F}{\sim} W_{1} \sim W_{2} \underset{F}{\sim} W_{3} \underset{t}{\sim} \cdots \sim_{t} W_{n} \underset{F}{\sim} y_{2}$.

If (1), then clearly $y_{1} \underset{H}{\sim} y_{2}$. If (2), then $y_{1} \underset{F}{\sim} U \in G_{1} \cup G_{2}$. This is impossible unless $y_{1}=1$ in which case $y_{2}=1$ and $y_{1} \underset{H}{{ }_{H}} y_{2}$.

We will next show that, for $V_{1}, V_{2} \in G, V_{1} \underset{G}{\sim} V_{2}$ if and only if $V_{1} \underset{L}{\sim} V_{2}$, thus showing that $L$ has unsolvable conjugacy problem. The statement from left to right is clear. Suppose that $V_{1} \sim V_{2}$. We may, without loss of generality, assume that $V_{1}$ and $V_{2}$ are $v$-reduced in $G$.

If $V_{1}, V_{2} \in H$, then either

(i) $V_{1} \sim V_{2}$; or

(ii) $V_{1} \underset{N}{\sim}\left(d^{m}\right.$ or $\left.e^{m}\right) \underset{v}{\sim}\left(e^{m}\right.$ or $\left.d^{m}\right) \underset{N}{\sim}\left(d^{k}\right.$ or $\left.e^{k}\right) \sim \cdots \sim V_{2}$. 
Using the fact that, for $y_{1}, y_{2} \in H, y_{1} \underset{N}{\sim} y_{2}$ implies $y_{1} \underset{H}{\sim} y_{2}$, both (i) and (ii) yield $V_{1} \underset{G}{\sim} V_{2}$. Now suppose $V_{1} \underset{L}{\sim} V_{2}$ and $V_{1}, V_{2} \in G-H$. If $V_{1} \underset{L}{\sim} V_{2}$ then there are cyclic permutations $V_{1}^{\prime}$ and $V_{2}^{\prime}$ of $V_{1}$ and $V_{2}$, respectively, with the same $v$-structures such that $d^{-k} V_{1}^{\prime} d^{k}=V_{2}^{\prime}$ or $e^{-k} V_{1}^{\prime} e^{k}=V_{2}^{\prime}$ for some $k$. Since all words involved are words of $G$ and since $G$ is embedded in $L$, one of the equalities must hold in $G$. Hence, $V_{1} \underset{G}{\sim} V_{2}$. Therefore, for $V_{1}, V_{2} \in G$, $V_{1} \underset{L}{\sim} V_{2}$ if and only if $V_{1} \underset{G}{\sim} V_{2}$ and so $L$ has unsolvable conjugacy problem.

Theorem 2. There is a finitely generated group $N$ with solvable conjugacy problem and an $H N N$-extension $L=\left\langle N, v ; v^{-1} d v=e\right\rangle$ where $d$ and $e$ are elements of $N$ of infinite order such that $L$ has unsolvable conjugacy problem.

Proof. Take $H$ and $G$ to be the infinitely generated groups of Theorem 1 and $N$ to be the group of Lemma 5 constructed from this $H$. By Lemma $6, L$ has unsolvable conjugacy problem. By Lemma 5 , to show that $N$ has solvable conjugacy problem it suffices to verify conditions (ii), (iii), and (iv). (Condition (i) is already known to be true.)

Condition (ii). Generators in $H$ are not equal to 1 in $H$ : If $i \notin$ range $f$, then $x_{i} \underset{H}{=} 1$ implies $x_{i} \underset{F r}{=} 1$ which is impossible. If $i=f(j)$, then

$$
x_{i} \underset{H}{=} 1 \Rightarrow z_{j} z_{1}^{-1} x_{1}^{j} z_{1} z_{j}^{-1} \underset{F r}{=} 1 \Rightarrow x_{1}^{j} \underset{F r}{=} 1 \Rightarrow j=0
$$

which is impossible. Therefore, $x_{i} \neq 1$ for all $i=1,2, \ldots$. Since $z_{i} \overline{\bar{H}} 1$ implies $z_{i}=1$, it is clear that $z_{i} \neq 1_{H}^{H}$ for all $i=1,2, \ldots$.

Condition (iii). Distinct generators of $H$ are not equal in $H$ : If $i, j \notin$ range $f$, then

$$
x_{i} \underset{H}{\bar{H}} x_{j} \Rightarrow x_{i} \underset{F r}{=} x_{j} \Rightarrow i=j .
$$

If $i \notin$ range $f$ and $j=f(k)$, then

$$
x_{i} \underset{H}{=} x_{j} \Rightarrow x_{i} \underset{F r}{=} z_{k} z_{1}^{-1} x_{1}^{k} z_{1} z_{k}^{-1}
$$

which is impossible. If $i, j \in$ range $f$, it is easy to see that $x_{i}=\bar{H}_{j}$ implies $i=j$. It is also easy to see that $z_{i} \underset{H}{\bar{H}} z_{j}$ implies $i=j$ and that $x_{i} \underset{H}{\bar{H}} z_{j}$ is impossible.

Therefore, distinct generators of $H$ are not equal in $H$.

Condition (iv). Given a word $W$ of $H$, we must decide if $W$ is a product of one or two generators or inverses of generators.

Semireduce $W$ to obtain $W^{\prime}$ (see proof of Lemma 2 for definition of semireduction). Then

(1) $W=\underset{\bar{H}}{=} z_{i_{1}}^{\varepsilon_{1}} z_{i_{2}}^{\varepsilon_{2}} \Leftrightarrow W^{\prime}=z_{i_{1}}^{\varepsilon_{1}} z_{i_{2}}^{\varepsilon_{2}}$.

(2) $W \underset{H}{=} x_{i}^{\varepsilon} z_{j}^{\delta} \Leftrightarrow W^{\prime} \underset{\overline{F r}}{=} x_{i}^{\varepsilon} z_{j}^{\delta}$ or $W^{\prime} \underset{\overline{F r}}{=} z_{k} z_{1}^{-1} x_{1}^{k} z_{1} z_{k}^{-1} z_{j}$ where $f(k)=i$.

(3) $W \underset{H}{=} x_{i}^{\varepsilon} x_{j}^{\delta}$ can be dealt with similarly. 
Therefore, $H$ satisfies conditions (ii), (iii), and (iv) of Lemma 5.

Theorem 3. There exist group presentations $N_{1}$ and $N_{2}$ each with a finite number of generators and a recursively enumerable set of defining relators and each having solvable conjugacy problem such that there is a free product with amalgamation $M$ of $N_{1}$ and $N_{2}$ with unsolvable conjugacy problem that amalgamates cyclic subgroups.

Proof. Let $N_{1}=\left\langle a, t ; r_{1}, r_{2}, \ldots\right\rangle$ and $N_{2}=\left\langle\hat{a}, \hat{t} ; \hat{r}_{1}, \hat{r}_{2}, \ldots\right\rangle$ be copies of the group $N$ of Theorem 2 . Recall that

$$
H=\left\langle x_{1}, z_{1}, x_{2}, z_{2}, \ldots ; z_{i}^{-1} x_{f(i)} z_{i}=z_{1}^{-1} x_{1}^{i} z_{1}(i=2,3, \ldots)\right\rangle .
$$

Let $\alpha$ and $\beta$ be the usual embeddings of $H$ into $N_{1}$ and $H$ into $N_{2}$, respectively. Let $W_{j}=\alpha\left(x_{j}\right)$ and $\hat{W}_{j}=\beta\left(x_{j}\right)$. Let

$$
M=\left\langle N_{1} * N_{2} ; W_{1}=\hat{W}_{1}\right\rangle .
$$

We claim that $W_{j} \underset{M}{\sim} \hat{W}_{j}$ if and only if $j \in \operatorname{range} f$. If $j \in \operatorname{range} f$, then $j=f(k)$ for some $k$ and

$$
\begin{aligned}
W_{j} & =\alpha\left(z_{k} z_{1}^{-1} x_{1}^{k} z_{1} z_{k}^{-1}\right) \underset{N_{1}}{\sim} \alpha\left(x_{1}^{k}\right) \underset{\bar{N}_{1}}{W_{1}^{k}} \overline{\bar{M}}^{k} \hat{W}_{1}^{k} \\
& =\beta\left(x_{1}^{k}\right) \underset{N_{2}}{\widetilde{N_{2}}} \beta\left(z_{k} z_{1}^{-1} x_{1}^{k} z_{1} z_{k}^{-1}\right)=\hat{W}_{j} .
\end{aligned}
$$

Therefore, if $j \in$ range $f$ then $W_{j} \dot{\sim} \hat{W}_{j}$.

Now suppose $W_{j} \widetilde{M} \hat{W}_{j}$. Since $M$ is a free product with amalgamation, there is a sequence of words $V_{1}, V_{2}, \ldots, V_{k}$ such that

$$
W_{j} \equiv V_{1} \underset{\alpha_{1}}{\sim} V_{2} \underset{\alpha_{2}}{\sim} V_{3} \underset{\alpha_{3}}{\sim} \cdots \underset{\alpha_{k-1}}{\sim} V_{k} \equiv \hat{W}_{j}
$$

where $V_{2}, \ldots, V_{k-1}$ are in the amalgamated part and the $\alpha_{i}$ alternate between $N_{1}$ and $N_{2}$. Therefore,

$$
\begin{aligned}
& W_{j} \underset{N_{1}}{\sim} W_{1}^{n} \text { for some } n \Rightarrow \alpha\left(x_{j}\right) \underset{N_{1}}{\sim} \alpha\left(x_{1}^{n}\right) \text { for some } n \Rightarrow \\
& \qquad x_{j} \underset{H}{\sim} x_{1}^{n} \text { for some } n \Rightarrow j \in \text { range } f .
\end{aligned}
$$

The second implication follows from the fact that, for $y_{1}, y_{2} \in H, y_{1} \underset{H}{y_{2}}$ if and only if $y_{1} \underset{N}{\sim} y_{2}$ (see proof of Lemma 6). Since $W_{j} \widetilde{M}_{j} \hat{W}_{j}$ if and only if $j \in$ range $f$ and range $f$ is nonrecursive, $M$ has unsolvable conjugacy problem.

\section{ProOF OF ASSERTIONS}

We now prove the assertions needed in Lemma 5.

(1) There is an algorithm to decide if elements of $G_{1} \cup G_{2}$ are conjugate in $N$. 
Proof. Suppose $U, V \in G_{1} \cup G_{2}$; then $U \underset{N}{\sim} V$ if and only if

(i) $U \underset{F}{\sim} V$; or

(ii) there are words $W_{1}, \ldots, W_{m}$ such that $U \underset{t}{\sim} W_{1} \underset{F}{\sim} W_{2} \sim W_{3} \sim \cdots \sim$ $W_{m} \sim V$.

Since (i) can be decided we need only consider (ii). By considering cyclic reductions with respect to * (i.e. with respect to the free product $F=H *\langle a, b\rangle$ ), it can be seen that if $1 \neq A \in G_{1}$ and $1 \neq B \in G_{2}$ then $A$ and $B$ are not conjugate in $F$. Therefore, $W_{2 k-1}$ and $W_{2 k}$ must be in the same $G_{i}$.

Suppose first that $U, V \in G_{1}$. Then $W_{1}, W_{2} \in G_{2}$ and $W_{3}, W_{4} \in G_{1}$. It can be seen that $U \underset{G_{1}}{\sim} W_{3}$ and $U \underset{G_{1}}{\sim} W_{4}$. Continuing in this fashion, we get

$$
U \underset{\langle a, b\rangle}{\sim} W_{4 k-1} \underset{\langle a, b\rangle}{\sim} W_{4 k} \text { for all } k=1, \ldots,[m / 4] .
$$

Since $V \in G_{1}, V$ is $W_{4 k-1}$ or $W_{4 k}$ for some $k$ and so $U \underset{\langle a, b\rangle}{\sim} V$. Therefore, (ii) occurs if and only if $U \sim \widetilde{F} V$ and so conjugacy in $N$ of elements of $G_{1}$ is decidable.

Now suppose $U, V \in G_{2}$. Then $W_{1} \in G_{1}, W_{m} \in G_{1}$, and $W_{1} \underset{N}{\sim} W_{m}$. By the above, $W_{1} \underset{\langle a, b\rangle}{\sim} W_{m}$. So, for $U, V \in G_{2}$, (ii) occurs if and only if $\phi^{-1}(U) \underset{\langle a, b\rangle}{\sim} \phi^{-1}(V)$.

The case $U \in G_{1}$ and $V \in G_{2}$ is similar.

(2) There is an algorithm to decide, given $V \in F$, if $V$ is conjugate in $F$ to an element of $G_{1} \cup G_{2}$ and, if so, to find such an element.

Proof. Let $V \in F$. We may assume that $V$ is cyclically reduced with respect to * . First (effectively) decide if $V \in\langle a, b\rangle$. If so, by assumptions (ii) and (iii), $V \sim U \in G_{1} \cup G_{2}$ if and only if $V \sim U \in G_{1}$ or $V \sim b^{m}$ which can be decided. If $V \notin\langle a, b\rangle$ then $V \sim U \in G_{1} \cup G_{2}$ if and only if $V \sim U \in G_{2}$. Suppose that $V \equiv A_{1} H_{1} \cdots A_{m} H_{m}$ where $A_{i} \in\langle a, b\rangle$ and $H_{i} \in H$. If $V \sim U \in G_{2}$ then by assumptions (ii) and (iii) each $A_{i}$ is a product of at most three words of the forms $a^{-j} b^{ \pm 1} a^{j}$ or $b^{k}$ ( $k^{-}$an integer). It can be effectively determined if each $A_{i}$ is such a product and, if so, each $A_{i}$ can be effectively written as such a product. The only possible cyclically reduced $U$ (up to cyclic permutations) can then be determined and tested.

(3) There is an effective procedure to determine, for arbitrary $X, Y \in F$, if there is a $Z \in G_{2}$ such that $X Z Y \in G_{1}$ and, if so, to produce the finite number of possible $Z$.

Proof. Suppose $X=A_{1} H_{1} \cdots A_{m} H_{m} A_{m+1}$ and $Y=B_{1} K_{1} \cdots B_{l} K_{l} B_{l+1}$ where $A_{i}, B_{i} \in\langle a, b\rangle$ and $H_{i}, K_{i} \in H$. Since $X Z Y \in G_{1}$, all $H$ factors must cancel and either

(a) $Z=A_{m+1}^{-1} H_{m}^{-1} A_{m}^{-1} \cdots H_{1}^{-1} Z^{\prime} K_{l}^{-1} B_{l}^{-1} \cdots K_{1}^{-1} B_{1}^{-1} \in G_{2}$ and $A_{1} Z^{\prime} B_{l+1}$ $\in G_{1}$; or 
(b) $Z=A_{m+1}^{-1} H_{m}^{-1} A_{m}^{-1} \cdots H_{j}^{-1} Z^{\prime} K_{q}^{-1} B_{q}^{-1} \cdots K_{1}^{-1} B_{1}^{-1} \in G_{2}$ where $2 \leq j \leq$ $m, 1 \leq q \leq l-1, Z^{\prime}$ is $h$-free and $A_{1} H_{1} \cdots A_{j-1} H_{j-1} A_{j} Z^{\prime} B_{q+1} K_{q+1}$ $\cdots B_{l} K_{l+1} B_{l+1} \in G_{1}$.

If (a) then $H_{1}^{-1}=h_{i_{1}}^{\delta_{1}} h_{i_{2}}^{\delta_{2}}$ and $K_{l}^{-1}=h_{j_{1}}^{t_{1}} h_{j_{2}}^{t_{2}}$ for some $\delta_{2}, t_{1}= \pm 1$ and $\delta_{1}, t_{2}=0$ or \pm 1 . By assumption (iv), there is an effective procedure to determine if $H_{1}^{-1}$ and $K_{l}^{-1}$ can be written as such and, if so, to so write them. Since $Z^{\prime}$ is $h$-free and $Z \in G_{2}$, there are four possibilities: $h_{i_{2}}^{\delta_{2}} Z^{\prime} h_{j_{1}}^{t_{1}} \in G_{2}$, $Z^{\prime} h_{j_{1}}^{t_{1}} \in G_{2}, h_{i_{2}}^{\delta_{2}} Z^{\prime} \in G_{2}, Z^{\prime} \in G_{2}$. Since $A_{1} Z^{\prime} B_{l+1} \in G_{1}$ implies that the exponent sum of $b$ in $A_{1} Z^{\prime} B_{l+1}$ is zero, each of these four possibilities give rise to finitely many possible $Z^{\prime}$ which in turn give rise to finitely many possible $Z$.

If (b) then $A_{j} Z^{\prime} B_{q+1} \underset{\langle a, b\rangle}{=} 1$ which implies that

$$
Z=A_{m+1}^{-1} H_{m}^{-1} A_{m}^{-1} \cdots H_{j}^{-1} A_{j}^{-1} B_{q+1}^{-1} K_{q}^{-1} B_{q}^{-1} \cdots K_{1}^{-1} B_{1}^{-1}
$$

again giving rise to only finitely many possible $Z$.

(4) If $X, Y \in F-G_{2}$, if $D \in G_{2}$ is reduced and if $X D Y \in G_{2}$, then $\#_{b}(D) \leq \#_{b}(X)+\#_{b}(Y)+2$.

Proof. Let $V \equiv v_{1} \cdots v_{m}$ where $v_{i} \in\left\{b^{ \pm 1},\left(h_{i} a^{-i} b a^{i}\right)^{ \pm 1}(i=1,2, \ldots)\right\}$. Suppose $V$ is freely reduced with respect to the free group $G_{2}$ and $\#_{b}(V) \geq 3$. It is easy to see that if $W$ is obtained from $V$ by freely reducing with respect to the free product $H *\langle a, b\rangle$, then the subword of $V$ containing the three $b$ can be uniquely recovered from $W$.

Suppose $D \in G_{2}, X D Y \in G_{2}$ and $\#_{b}(D) \geq \#_{b}(X)+\#_{b}(Y)+3$. Assume further that $X, D$, and $Y$ are reduced with respect to *. It suffices to show that $X \in G_{2}$. Consider the cancellation that occurs in the product $X D Y$. Let

$$
X \equiv X_{1} X_{2} ; D \equiv X_{2}^{-1} D_{1} Y_{1}^{-1} \text {; and } Y \equiv Y_{1} Y_{2} \text {. }
$$

We have that $X_{1} D_{1} Y_{2} \in G_{2}$ is reduced with respect to $*$ and $\#_{b}\left(D_{1}\right) \geq 3$. By the comment above, the shortest words $X_{1}^{\prime \prime}$ and $Y_{2}^{\prime}$ such that $X_{1} \equiv X_{1}^{\prime} X_{1}^{\prime \prime}, Y_{2} \equiv$ $Y_{2}^{\prime} Y_{2}^{\prime \prime}$ and $X_{1}^{\prime \prime} D_{1} Y_{2}^{\prime} \in G_{2}$ can be effectively found. Since $X_{1}^{\prime \prime}$ and $Y_{2}^{\prime}$ are unique, $X_{1}^{\prime} X_{1}^{\prime \prime} D_{1} Y_{2}^{\prime} Y_{2}^{\prime \prime} \in G_{2}$, and $X_{1}^{\prime \prime} D_{1} Y_{2}^{\prime} \in G_{2}$, it follows that $X_{1}^{\prime}$ and $Y_{2}^{\prime \prime} \in G_{2}$. Since $X_{1}^{\prime \prime}$ is the shortest word such that $X_{1}^{\prime \prime} D_{1} Y_{2}^{\prime} \in G_{2}$ and since $X_{2}^{-1} D_{1} Y_{1}^{-1} \in G_{2}$, it follows that $X_{2}^{-1}$ ends in $X_{1}^{\prime \prime}$. That is, $X_{2}^{-1} \equiv X_{3} X_{1}^{\prime \prime}$ and $X_{3} \in G_{2}$. Therefore, $X \equiv X_{1}^{\prime} X_{1}^{\prime \prime} X_{2}=X_{1}^{\prime} X_{3}^{-1} \in G_{2}$ since $X_{1}^{\prime} \in G_{2}$ and $X_{3} \in G_{2}$.

\section{Conclusion}

The existence of a finitely presented group $H$ and an HNN-extension $G=$ $\left\langle H, t ; t^{-1} a t=b\right\rangle$ where $H$ has solvable conjugacy problem but $G$ has unsolvable conjugacy problem is open. The analogous question for free products with amalgamation is also open. 


\section{REFERENCES}

1. M. Anshel and P. Stebe, The solvability of the conjugacy problem for certain HNN groups, Bull. Amer. Math. Soc. 80 (1974), 266-270.

2. L. P. Comerford and B. Truffault, The conjugacy problem for free products of sixth-groups with cyclic amalgamation, Math. Z. 149 (1976), 169-181.

3. R. D. Hurwitz, On cyclic subgroups and the conjugacy problem, Proc. Amer. Math. Soc. 79 (1980), 1-8.

4. S. Lipschutz, The conjugacy problem and cyclic amalgamations, Bull. Amer. Math. Soc. 81 (1975), 114-116.

Department of Mathematics, Indiana University at South Bend, South Bend, Indiana 46634

Current address: Department of Mathematics, United States Naval Academy, Annapolis, Maryland 21402 